\title{
The Association between Awareness of Sexual Behavior and Cognitive Social Capital among High School Students in Vietnam
}

\author{
Kaori Watanabe' ${ }^{1}$, Yoshihiro Kaneko ${ }^{2}$, Koji Fujita², Yutaka Motohashi2 \\ ${ }^{1}$ Japanese Red Cross Akita College of Nursing, Akita, Japan \\ ${ }^{2}$ Department of Public Health, Akita University School of Medicine, Akita, Japan \\ Email: kaori-w@rcakita.ac.jp
}

Received 27 January 2014; revised 28 February 2014; accepted 11 March 2014

Copyright (C) 2014 by authors and Scientific Research Publishing Inc.

This work is licensed under the Creative Commons Attribution International License (CC BY). http://creativecommons.org/licenses/by/4.0/

c. (i) Open Access

\begin{abstract}
Background: Globally, adolescent sexual behavior is a major health concern and the focus of many researches. However, negative attitude towards premarital sex and sense of community belonging have not been examined in relation to adolescent sexual behavior. The purpose of this study was to examine whether these two factors are associated with adolescent sexual behavior. Methods: We conducted a cross-sectional survey of 1672 eleventh grade students from three public high schools in Hanoi, Vietnam. Participants' sense of community belonging and negative attitude towards premarital sex were assessed through a self-administered questionnaire. We performed multiple logistic regression analyses to examine associations between the cognitive social capital concepts, such as a sense of community belonging. Results: $92.3 \%$ of students agreed with a sense of community belonging, but only $14.1 \%$ of students accepted premarital sex. A high sense of community belonging was significantly associated with negative attitude towards premarital sex OR (95\% CI), male 3.16 (2.3 - 4.34), female 8.77 (5.38 - 14.29). After adjusting for a sense of community belonging, the association disappeared between acceptance of premarital sex and sexual experience of peers, proper time to wear a condom, and Rosenberg Self-Esteem Scale score. Conclusion: Cognitive social capital, such as sense of community belonging, was associated with negative attitude towards premarital sex. Therefore, cognitive social capital concepts should be included in sexual behavior and health promotions, especially with adolescents.
\end{abstract}

\section{Keywords}

Social Capital; Sexual Behavior; Adolescent; Reproductive Health; Self-Esteem; Vietnam

How to cite this paper: Watanabe, K., Kaneko, Y., Fujita, K. and Motohashi, Y. (2014) The Association between Awareness of Sexual Behavior and Cognitive Social Capital among High School Students in Vietnam. Open Journal of Preventive Medicine, 4, 116-122. http://dx.doi.org/10.4236/ojpm.2014.43016 


\section{Introduction}

Globally, adolescent sexual behavior, especially premarital sex, is a growing public health concern because of its connection to adolescent pregnancy and sexually transmitted diseases. For example, pregnant adolescent girls face a much higher risk of maternal mortality as their immature bodies are unable to cope with the experience. Another serious risk is unsafe abortion, which causes the death of many adolescent girls and injures [1]-[3].

In Vietnam, premarital or unprotected sexual intercourse is a growing health and social concern. The country's dramatic economic growth and political changes have resulted in social changes, including premarital sex attitudes and behaviors. Although premarital sex is long considered as a social taboo, the government has branded it as a "social evil" and believes that high rates of premarital sexual activities are widespread among the younger generation [4]. Along with structural economic and political changes resulting from the country's doi moi (renovation), the increased presence of foreign media is often mentioned as a leading cause of unhealthy social changes [4]. The foreign images, attitudes, and ideas that Vietnamese youths are exposed through the Internet, imported movies, pornography, and sexualized music, are considered influential and unwholesome information [5] [6] and the cause of increased sexual permissiveness, premarital sexual activity, sexually transmitted infections, unintended pregnancy, and abortion [4].

According to a Survey Assessment of Vietnamese Youth (SAVY), the largest and the most comprehensive survey of youth in Vietnam, the mean age that a youth (aged 14 to 25 years) first experiences sexual intercourse had decreased from 19.6 years old in 2003 to 18.1 years old in 2008, and the rate of premarital sex increased from $7.6 \%$ in 2003 to 9.5\% in 2008 [7] [8]. Ghuman et al.'s (2006) research also reported that premarital sex was becoming more common in Vietnam [9].

Sexual activity is often initiated between the ages of 15 and 22 [10], when many adolescents are still at school, and more than half (51.2\%) of new HIV infections are among 15 to 24 years old [11]. Therefore, it is necessary to address the sexual behaviors of high school students. A considerable number of demographic and reproductive health studies in Vietnam have been published in recent years [10], but few studies have focused on the sexual awareness and sexual behavior of Vietnamese high school students.

Of these studies, one study focused on HIV/AIDS preventive behavior [12], and most previous studies have focused on raising self-esteem and sex education as important intervention techniques that influence sexual awareness and behavior of adolescents [13]. However, recent research has suggested that cognitive social capital, such as a sense of community belonging, might influence behavior modification [14] [15]. In their systematic review of fifteen studies, McPherson et al. explored the role and impact of social capital on sexual health outcomes [16]-[22]. The evidence suggested that social capital is a protective factor for sexual health behaviors [22] [23].

Based on reports showing that low-levels of social capital increased the risk of unhealthy sexual behavior [22], we hypothesized that the sexual attitudes of Vietnamese high school students' permissiveness and acceptance of premarital sex, are influenced by cognitive social capital, such as a sense of community belonging. Thus, the purpose of this cross-sectional study was to examine whether cognitive social capital is associated with raised awareness of sexual behaviors among Vietnamese high school students.

\section{Methods}

The participants of this study were selected from eleventh grade students at three public high schools in Hanoi, the capital city of the Socialist Republic of Vietnam [16] [17], which covers an area of $3323 \mathrm{~km}^{2}$ and has a population 6.844 million (Vietnam General Statistics Office-GSO, 2012). The Center for Population Information and Documentation (CPID), the General Office for Population and Family Planning (GOPFP), and the Ministry of Health of Vietnam also supported the survey. We conducted a questionnaire-based survey from September to October, 2012, with 1672 students participating. Responses were received from 1672 students, a response rate of 100\%. Complete data was received from 1653 respondents (98.9\%), consisting of 896 males (54.2\%) and 757 females (45.8\%). Incomplete surveys were excluded from the analysis.

The typical school system in Vietnam comprises elementary school (lasting five years), junior high school (four years), senior high school (three years), and university (four years). As education is compulsory only for the nine years of elementary and junior high school, the high school enrollment rate drops to 63.2\% (2011-2012 Ministry of Education and Training). Owing to the shortage of school buildings and teachers, many schools in 
Vietnam have morning and afternoon sessions.

The survey was a self-administrated questionnaire, and all items and related documents were first written in English then translated into Vietnamese. The native GOPFP staff proofread the materials. We obtained permission to conduct the survey from the appropriate person in charge at each school. The high school staff and GOPFP distributed the questionnaires, and explained the details to participating students. The survey was anonymous. Each student sealed the completed survey in an envelope to protect their privacy.

The questionnaire assessed the participants' sexual knowledge, awareness of sexual behavior, awareness of peers' sexual intercourse experience, cognitive social capital, and used the Rosenberg Self-Esteem Scale score.

We assigned each variable to groups such as 1) male or female for gender, 2) whether the respondent agreed or not with the questions on social capital and sexual awareness, or 3) correct or incorrect answers to the sexual knowledge questions. Then, we compared the means of the two groups using the independent $t$-test, and performed multiple logistic-regression analysis to examine the association between acceptance of premarital sex and social capital with adjustment for Rosenberg Self-Esteem Scale scores (divided into two groups with the median), wearing a condom, and sexual experience of peers (model 1). This was followed by analysis of the associations adjusted for sense of community belonging, the Rosenberg Self-Esteem Scale scores, wearing a condom, and sexual experience of peers (model 2).

All analyses were computed using SPSS 17 (Chicago, IL) statistical software.

This study was approved by the Ethical Review Board of the Japanese Red Cross Akita College of Nursing (approval number 24-003). We had the cooperation of the high schools and obtained written informed consent from the participating students.

\section{Results}

Of the 1653 respondents with complete data, 99.9\% were 16 - 18 years old and most were 16 years old. Most respondents (86.7\%) were from the nuclear families.

Table 1 shows the sexual knowledge of the participants. The percentage of the participants who correctly answered the question on the proper time to wear a condom was 53.3\%. Table 1 also shows the results of sense of community belonging and awareness of sexual behavior. Only $14.1 \%$ of students accepted premarital sex and the percentage of those who accepted premarital sex was higher in males than females, but $70.2 \%$ of the participants knew peers with sexual intercourse experience. As to social capital, 92.3\% agreed with a sense of community belonging.

Table 2 shows the Rosenberg Self-Esteem Scale scores. The overall mean score was $18.0 \pm 3.9$ (not indicated in the Table 2), and males scored higher than females. The overall mean score of participants was lower compared with reported scores from similar populations in the USA or Europe.

Respondents who agreed with the item "we should not have premarital sex" had a higher Rosenberg Self-Esteem score than those who disagreed. Also the score was higher with participants who agreed with a sense of community belonging than with participants who disagreed.

Table 3 shows the results of the multiple logistic regression analyses, including sense of community belonging as a variable. There was a positive association between sense of community belonging and acceptance of premarital sex even after adjusting for the Rosenberg Self-Esteem Scale score, sexual knowledge, and sexual experience of peers. In model 1, the acceptance of premarital sex was significantly associated with Rosenberg Self-Esteem Scale score, wearing a condom, and sexual experience of peers. In model 2, after adjusting for a sense of community belonging, the association disappeared between acceptance of premarital sex and the Rosenberg Self-Esteem Scale score, and wearing a condom, but a significant association was found between acceptance of premarital sex and sense of community belonging OR (95\% CI), male 3.16 (2.3 - 4.34), female 8.77 (5.38 - 14.29).

\section{Discussion}

Our results indicate that sexual knowledge and self-esteem are explanatory factors relevant to the risk of sexual behaviors when a sense of community belonging is not included in a logistic regression model. This coincides with previous research that reported adequate sexual knowledge and high level of self-esteem were factors associated with the risk of adolescent sexual behaviors. However, when a sense of community belonging was included in the logistic regression model, the association was attenuated, and we found only a sense of community 
Table 1. Sexual knowledge, sense of community belonging and sexual awareness $(n=1653)$.

\begin{tabular}{|c|c|c|c|c|c|c|}
\hline \multirow{2}{*}{ Variable } & \multicolumn{2}{|c|}{ Overall $(n=1653)$} & \multicolumn{2}{|c|}{ Male $(n=896)$} & \multicolumn{2}{|c|}{ Female $(n=757)$} \\
\hline & $n$ & $\%$ & $n$ & $\%$ & $n$ & $\%$ \\
\hline \multicolumn{7}{|l|}{ 1) Sexual Knowledge } \\
\hline Proper time to wear a condom & 1454 & & 851 & & 603 & \\
\hline Correct answer & 775 & 53.3 & 497 & 58.4 & 278 & 46.1 \\
\hline Incorrect answer & 679 & 46.7 & 354 & 41.6 & 325 & 53.9 \\
\hline \multicolumn{7}{|l|}{ 2) Sense of Community Belonging and Sexual Awareness } \\
\hline We should not have premarital sex & 1646 & & 891 & & 755 & \\
\hline Agree & 1414 & 85.9 & 714 & 80.1 & 700 & 92.7 \\
\hline Disagree & 232 & 14.1 & 177 & 19.9 & 55 & 7.3 \\
\hline I have sense of community belonging & 1646 & & 892 & & 754 & \\
\hline Agree & 1519 & 92.3 & 813 & 91.1 & 706 & 93.6 \\
\hline Disagree & 127 & 7.7 & 79 & 8.9 & 48 & 6.3 \\
\hline Most of my peers (siblings and friends) have sexual experience & 1620 & & 883 & & 73 & \\
\hline Agree & 1137 & 70.2 & 605 & 68.5 & 532 & 72.2 \\
\hline Disagree & 483 & 29.8 & 278 & 31.5 & 205 & 27.8 \\
\hline
\end{tabular}

Table 2. Rosenberg self-esteem scale score.

\begin{tabular}{lccc}
\hline \multicolumn{1}{c}{ Variable } & $\boldsymbol{n}$ & Average (SD) & P value \\
\hline Gender & & & \\
Male & 853 & $18.2(4.0)$ & 0.01 \\
Female & 707 & $17.7(3.7)$ & 0.04 \\
We should not have premarital sex & & $17.9(3.8)$ & $<0.01$ \\
Agree & 1330 & $18.5(4.3)$ & \\
Disagree & 223 & $18.3(3.8)$ & $14.8(4.1)$ \\
Sense of community belonging & 1436 & $18.1(3.9)$ & 0.55 \\
Agree & 119 & $17.9(3.8)$ \\
Disagree & & & \\
Proper timing to wear condom & 732 & 654 & \\
Correct answer & & & \\
Incorrect answer &
\end{tabular}

belonging significantly associated with the risk of sexual behaviors. This implies that, at least among adolescents in developing countries like Vietnam, social capital is a more important factor associated with the risk of sexual behaviors compared with sexual knowledge and self-esteem.

This finding is in line with a considerable number of studies on the association between social capital and the risk of sexual behaviors published in the US or other countries [16]-[22]. These concluded that social capital decreases the risk of sexual behaviors.

Under the influence of Confucianism, a conservative influence in society, Vietnam retains traditional orientations towards family and community [24]. Its people largely adhere to the principles of Confucian culture, connectedness to one's family and community, ensuring harmony and order in the family and community, and abiding them [25] [26]. These concepts seem to be similar to the cognitive social capital concept in the western world.

According to a previous study of influences on traditional Confucian culture and adolescents' sexual behaviors in three Asian cities, including Hanoi, the traditional Confucian cultural norms were noticeably stronger in Hanoi than other cities. Prevalence of sexual coitus among adolescents and young adults was lowest in Hanoi, 
Table 3. Association between acceptability of premarital sex, social capital, and sexual knowledge $(n=1653)$.

\begin{tabular}{|c|c|c|c|c|c|c|c|c|}
\hline \multirow{3}{*}{ Variables } & \multicolumn{4}{|c|}{ Model 1} & \multicolumn{4}{|c|}{ Model 2} \\
\hline & \multicolumn{2}{|l|}{ Male } & \multicolumn{2}{|l|}{ Female } & \multicolumn{2}{|l|}{ Male } & \multicolumn{2}{|l|}{ Female } \\
\hline & OR (95\% CI) & $\boldsymbol{P}$ & OR (95\% CI) & $\boldsymbol{P}$ & OR $(95 \% \mathrm{CI})$ & $\boldsymbol{P}$ & OR $(95 \% \mathrm{CI})$ & $\boldsymbol{P}$ \\
\hline \multicolumn{9}{|l|}{ Rosenberg self-esteem scale score } \\
\hline $18-30$ & $\begin{array}{c}2.15 \\
(1.64-2.81)\end{array}$ & $<0.01$ & $\begin{array}{c}7.63 \\
(4.59-12.7)\end{array}$ & $<0.01$ & $\begin{array}{c}1.05 \\
(0.75-1.48)\end{array}$ & 0.78 & $\begin{array}{c}1.63 \\
(0.86-3.08)\end{array}$ & 0.13 \\
\hline $0-17$ & ref & & ref & & ref & & ref & \\
\hline \multicolumn{9}{|l|}{ Proper timing to wear a condom } \\
\hline Correct answer & $\begin{array}{c}1.61 \\
(1.24-2.1)\end{array}$ & $<0.01$ & $\begin{array}{c}4.82 \\
(3.05-7.64)\end{array}$ & $<0.01$ & $\begin{array}{c}0.93 \\
(0.67-1.27\end{array}$ & 0.64 & $\begin{array}{c}1.27 \\
(0.72-2.21)\end{array}$ & 0.41 \\
\hline Incorrect answer & ref & & ref & & ref & & ref & \\
\hline \multicolumn{9}{|l|}{ Sense of community belonging } \\
\hline Strongly agree, agree & & & & & $\begin{array}{c}3.16 \\
(2.3-4.34)\end{array}$ & $<0.01$ & $\begin{array}{c}8.77 \\
(5.38-14.29)\end{array}$ & $<0.01$ \\
\hline Disagree, strongly disagree & & & & & ref & & & \\
\hline \multicolumn{9}{|l|}{ Sexual experience of peers } \\
\hline Disagree & $\begin{array}{c}3.12 \\
(2.16-4.53)\end{array}$ & $<0.01$ & $\begin{array}{c}4.33 \\
(2.27-8.28)\end{array}$ & $<0.01$ & $\begin{array}{c}2.06 \\
(1.39-3.05)\end{array}$ & $<0.01$ & $\begin{array}{c}1.67 \\
(0.83-3.36)\end{array}$ & 0.15 \\
\hline Agree & ref & & ref & & ref & & ref & \\
\hline
\end{tabular}

OR: Odds Ratio; CI: Confidence Interval. Results are based on logistic regression analysis. Model 1 has been adjusted for Rosenberg Self-Esteem Scale Score, Proper Timing to Wear Condom and Most of Peer Have Sexual Experience. Model 2 has been adjusted for Rosenberg Self-Esteem Scale Score, Proper Timing to Wear Condom, Community Belonging and Most of Peer Have Sexual Experience.

and Vietnamese adolescents with more traditional views were less likely to engage in sexual activity [25] [26]. This finding suggested that Confucian values may have a distinct association with adolescents' sexual behaviors, and that social capital, in general, is associated with less risky sexual behavior [22] [23]. Also, throughout Vietnam, traditional Confucian values regard virginity as a virtue among both males and females [5].

Cultural environment change is an important factor influencing adolescents' sexual behaviors. In the wake of modernization, rapid industrialization, and contemporary globalization, Vietnam is undergoing marked sociocultural changes, which are weakening aspects of traditional Confucian values, including dependence and connectedness to the family and community.

A possible reason that sexual knowledge and self-esteem were weaker factors than sense of community belonging, compared with previous studies, may be that this study simultaneously analyzed sexual knowledge and self-esteem with social capital. Future studies of adolescent sexual behaviors should consider the social capital factors.

The findings of this study highlight the importance of strengthening education to increase a sense of community belonging, and sexual and reproductive health knowledge among Vietnamese high school students.

Several study limitations need to be considered. First, the cross-sectional nature of this survey design does not allow for changes in participants' beliefs, past, present and future, to be examined. This can be achieved with a long-term longitudinal study. Second, because this is a self-administered questionnaire survey, there is a possibility of reporting bias.

\section{Conclusion}

Cognitive social capital, such as a sense of community belonging, was found to be associated with a negative attitude towards premarital sex. This suggests that cognitive social capital should be included in sexual health promotion, especially with adolescents.

\section{Acknowledgements}

We are grateful for the collaboration of staff members from the Ministry of Health Center for Population Infor- 
mation and Documentation (CPID), General Office for Population and Family Planning (GOPFP) of Socialist Republic of Vietnam, and participating high school staff members, students, and their parents/guardians in conducting this study.

\section{References}

[1] Anthony, D. and United Nations Children's Fund (UNICEF) (2011) The State of the World's Children 2011—Adolescence: An Age of Opportunity. http://www.unicef.org/sowc2011/pdfs/SOWC-2011-Main-Report_EN_02092011.pdf

[2] UNFPA (2013) The State of World Population 2013 Motherhood in Childhood-Facing the Challenge of Adolescent Pregnancy.

[3] World Health Organization (WHO) (2008) Unsafe Abortion: Global and Regional Estimates of the Incidence of Unsafe Abortion and Associated Mortality in 2008. 6th Edition, WHO, Geneva, 2011.

[4] Jayakody, R., Heckert, J. and Anh, D. (2010) Social Change and Premarital Sexual Behavior and Attitudes in Vietnam. SAGE Publications Ltd., Thousand Oaks.

[5] Linh, L. and Blum, R. (2009) Premarital Sex and Condom Use among Never Married Youth in Vietnam. International Journal of Adolescent Medicine and Health, 21, 299-312.

[6] Ngo, A., Ross, M. and Ratliff, E. (2008) Internet Influence on Sexual Practice among Young People in Hanoi, Vietnam. Culture, Health \& Sexuality, 10, S201-S213. http://dx.doi.org/10.1080/13691050701749873

[7] Ministry of Health, General Statistic Office, World Health Organization and the United Nations Children Fund (2005) Survey Assessment of Vietnamese Youth Round 1 (SAVY 1). Hanoi.

[8] Ministry of Health, General Statistic Office, World Health Organization and the United Nations Children Fund (2010) Survey Assessment of Vietnamese Youth Round 2 (SAVY 2). Hanoi.

[9] Ghuman, S., Loi, V., Huy, V. and Knodel, J. (2006) Continuity and Change in Premarital Sex in Vietnam. International Family Planning Perspectives, 32, 166-174. http://dx.doi.org/10.1363/3216606

[10] Mensch, B., Clark, W. and Dang, N. (2002) Premarital Sex in Vietnam: Is the Current Concern with Adolescent Reproductive Health Warranted? Population Council No.163. http://www.popcouncil.org/pdfs/wp/163.pdf

[11] van Wijingaarden, J. (2006) UNICEF Consultant Exploring Factors and Processes Leading to HIV Risk among the Most Vulnerable Children and Adolescent in Vietnam.

[12] Taechaboonsermsak, P., Tuan, L. and Apinuntavech, S. (2008) Factors Associated with HIV/AIDS Preventive Behavior among High School Students in Dongda District, Hanoi, Vietnam. Journal of Public Health, 38, 174-185.

[13] Goodson, P., Buhi, E.R. and Duncsmore, S. (2006) Self-Esteem and Adolescent Sexual Behaviors, Attitudes, and Intentions: A Systematic Review. Journal of Adolescent Health, 38, 310-319. http://dx.doi.org/10.1016/j.jadohealth.2005.05.026

[14] Kawachi, I., Subramanian, S. and Kim, D. (2008) Social Capital to Kenkou. Hyoron Sha Co., Ltd., Tokyo, 82-97.

[15] Kawachi, I., and Berkman, L. (2000) Social Cohesion, Social Capital, and Health. In: Berkman, L.F. and Kawachi, I. Eds., Social Epidemiology, Oxford University Press, New York, 174-190.

[16] McPherson, K., Kerr, S., McGee, E., Cheater, F. and Morgan, A. (2013) The Role and Impact of Social Capital on the Health and Wellbeing of Children and Adolescents: A Systematic Review. Glasgow Centre for Population Health. http://www.gcph.co.uk/assets/0000/3647/Social capital final 2013.pdf

[17] Harris, L. (2007) Association between Youth Assets and Sexual Activity: Does Adult Supervision Play a Role? Child Care Health and Development, 33, 448-454. http://dx.doi.org/10.1016/j.jadohealth.2005.05.026

[18] Daniel, W. (2006) Parental Influence on Young People’s Sexual Behavior: A Longitudinal Analysis. Journal of adolescence, 29, 474-494.

[19] Oman, R. (2005) Youth Assets and Sexual Risk Behavior: The Importance of Assets for Young Residing in One- Parent Households. Perspectives on Sexual and Reproductive Health, 37, 25-31. http://dx.doi.org/10.1363/3702505

[20] Evans, A. (2004) An Exploration of the Relationship between Youth Assets and Engagement in Risky Sexual Behaviors. Journal of Adolescent Health, 35, 424.e21-424.e30.

[21] Oman, R., Vesely, S., et al. (2004) The Association between Multiple Youth Assets and Sexual Behavior. American Journal of Health Promotion, 19, 12-18. http://dx.doi.org/10.4278/0890-1171-19.1.12

[22] Crosby, R. (2003) Social Capital as a Predictor of Adolescents' Sexual Risk Behavior: A State-Level Exploratory Study. AIDS and Behavior, 7, 245-252. http://dx.doi.org/10.1023/A:1025439618581

[23] Agardh, A., et al. (2010) Social Capital and Sexual Behavior among Ugandan University Students. Global Health Action, 3, 5432. http://dx.doi.org/10.3402/gha.v3i0.5432 
[24] Dalton, R., et al. (2002) Social Relations and Social Capital in Vietnam the 2001 World Values Survey. Comparative Sociology.

[25] Gao, E., Zuo, X., Wang, L., Lou, C., Cheng, Y. and Zabin, L. (2012) How Does Traditional Confucian Culture Influence Adolescents' Sexual Behavior in Three Asian Cities? Journal of Adolescent Health, 50, S12-S17. http://dx.doi.org/10.1016/j.jadohealth.2011.12.002

[26] Malik, A.S., Boyko, O., Atkar, N. and Young, W.F. (2001) A Comparative Study of MR Imaging Profile of Titanium Pedicle Screws. Acta Radiologica, 42, 291-293. http://dx.doi.org/10.1080/028418501127346846 